\title{
Congenital complete agenesis of pericardium
}

INSERM

\section{Source}

INSERM. (1999). Orphanet: an online rare disease and orphan drug data base. Congenital complete agenesis of pericardium. ORPHA:99129

Congenital complete agenesis of pericardium is a rare, mostly asymptomatic, congenital heart malformation characterized by the complete absence of the entire pericardium, or by the absence of either the right (uncommon) or left pericardium. It is occasionally associated with chest pain (common), dyspnea, dizziness, bradycardia and syncope, while exertional manifestations are rare. The disease is usually incidentally diag nosed during surgery or at autopsy. 